\title{
ON THE 'THEORY OF SERIES WITH FUNCTION TERMS
}

\author{
TAMOTSU TSUCHIKURA
}

(Received July 12, 1950)

Introduction. In the theory of series we find many theorems concerning the divergence nature of the series, for example the classical theorem of Abel-Dini, the theorem of Agnew on the rearrangement of terms [?] and the following:

THEOREM A. (J. D. HILL[3]) If a' series' $\Sigma a_{i}$ is contitionally convergent, then for all $x \in(0,1)$ except for a set of the first category, wo have

$$
\liminf _{n \rightarrow \infty} \sum_{i=1}^{n} \beta_{i} a_{i}=-\infty, \quad \limsup _{n \rightarrow \infty} \sum_{i=1}^{n} \beta_{i} a_{i}=+\infty,
$$

where $\beta_{i}$ are the coefficients of dyadic expansion of $x$.

THEOREM B. (R. P. AGNEW [1]). If a series $\Sigma a_{i}$ is not absolutely convergent, then there exists an increasing sequense of positive integers $\left\{n_{i}\right\}$ such that $n_{i}-n_{i-1} \rightarrow \infty$ as $i \rightarrow \infty$ and the series

diverges.

$$
\sum_{i=1}^{\infty} a_{n_{i}}
$$

In this paper we shall aim somewhat further, and generalize some of these theorems to the series of functions discussing simultaneously the nature of the category as in Theorem A. We shall prove in the first two $\S \S$ some theorems on functionals and in $\S 3$ we shall treat the series of functions as application of $\S \S 1$ and 2. The main pattern of the proof of Theorem 1 and 2 is due to S. Saks [4].

1. Let $\left\{S_{n}(t)\right\}$ be a sequence of measurable functions defined on a measurable set $I \subset(-\infty, \infty)$. The sequence $\left\{S_{n}(t)\right\}$ is called bounded in measure on $I^{1)}$, if, for every $\mu>0$ there exists a positive number $N=N(\eta)$ such that

$$
\text { meas } \underset{t}{\mathrm{E}}\left[t \in I ; \sup _{n}\left|S_{n}(t)\right|>N\right]>\eta \text {. }
$$

The sequence $\left\{S_{n}(t)\right\}$ is called to have the property $B(\varepsilon)$ on $I^{1)}$, if there is a positive number $N$ such that

$$
\text { meas } \underset{t}{\mathrm{E}}\left[t \in I ; \sup _{n}\left|S_{n}(t)\right|>N\right]<\varepsilon .
$$

In this and the next $\S \S$ we assume that: $(\alpha)$ meas $I<\infty ;(\beta) E$ is a metric space of the second category which consists of sequences of real numbers, and if $x_{0}=\left\{\beta_{i}\right\} \in E$ and $\left\{\gamma_{i}\right\} \in E$ then for any integer $j, x_{j}=$

1) 'This definition is different from Saks' [4]. 
$\left\{\beta_{1}, \cdots, \beta_{j}, \gamma_{j+1}, \gamma_{j+2}, \cdots\right\} \in E$ and $x_{j} \rightarrow x_{j}$ as $j \rightarrow \infty$. For $x=\left\{\beta_{i}\right\} \in E$ and $y=\left\{\gamma_{i}\right\} \in E$ if $\beta_{i}=\gamma_{i}$ for every $i>j$, then $x$ and $y$ are called $j$-equal.

Let $\left\{T_{k}\right\}(k=1,2, \ldots)$ be a sequence of measurable sets in $I$ such that the totality of the characteristic functions of $T_{k}$ forms an everywhere dense set in the space of the measurable characteristic functions, namely, if $J \subset I$ is a measurable set, then for any $\delta>0$ there exists an integer $k=k(\delta)$ such that

$$
\text { meas }\left(J-J T_{k}\right)+\text { meas }\left(T_{k}-T_{k} J\right)<\delta \text {. }
$$

THEOREM 1. Let $S_{n}(x, t)(n=1,2, \ldots)$ be real valued functions defined for all $x \in E$ and $t \in I$, measurable in $t$ and further satisfy the following properties :

(a) $S_{n}(x, t)$ is continuous in $x$ for any fixed $n$ and $t$;

(b) If $x$ and $x^{\prime}$ in $E$ are $j$-equal, then for any $\delta>0$ there correspond $a$ set $J \subset I$ and positive number $K$ depending on $j$ and $\delta$ such that

and

$$
\left|S_{n}(x, t)-S_{n}\left(x^{\prime}, t\right)\right|<K \quad(n=1,2, \cdots ; t \in J)
$$

$$
\text { meas } J>\text { meas } I-\delta \text {. }
$$

Unter these assumptions there exists a measurable set $A \subset I$ such that

(i) $\left\{S_{n}(x, t)\right\}$ is bounded in measure on $A$ for all $x \in E$;

and (ii) for all $x \in E$ except perhaps for a set of the first category, $\left\{S_{n}(x, t)\right\}$ is not bounded in measure on any set in $I-A$ of positive measure.

PROOF. Let $\alpha_{0}$ be the least upper bound of $\alpha>0$ for which there exists a set $H(\alpha) \subset E$ of the second category such that $\left\{S_{n}(x, t)\right\}$ is bounded in measure on a set of measure $>\alpha$ for all $x \in H(\alpha)$. If there is no such $\alpha_{0}$, then the set $A=0$ will be the required; hence we suppose that $\alpha_{0}>0$.

For any positive integer $q$, we shall show first that there exist a set $H_{q} \subset E$ of the second categiry and a set $P_{q} \subset I$ of measure $>\alpha_{0}-1 / q^{2}$ such that $\left\{S_{n}(x, t)\right\}$ has the property $B\left(1 / q^{2}\right)$ on $P_{q}$ for all $x \in H_{1}$, that is, for a suitable constant $M_{q}>0$,

$$
\text { meas } \underset{t}{\mathrm{E}}\left[t \in P_{q} ; \sup _{n}\left|S_{n}(x, t)\right| \geqq M_{q}\right]<\frac{1}{q^{2}} \quad\left(x \in H_{q}\right) .
$$

To $x \in H\left(\alpha_{0}-1 / q^{2}\right)$ there correspond a set $T_{k}(k=k(x))$ and a positive integer $m=m(x)$ such that

$$
\text { meas } \underset{t}{\mathrm{E}}\left[t \in T_{k} ; \sup _{n}\left|S_{n}(x, t)\right| \geqq m\right]<1 / q^{2} \text {, }
$$

$$
\text { meas } T_{k}>\alpha_{0}-1 / q^{2} \text {. }
$$

Let $H_{1, k}, m$, denote the set of all $x \in H\left(\alpha_{3}-1 / q^{2}\right)$ for which (1.2) holds for fixed $k$ and $m$. Since $H\left(\alpha_{0}-1 / q^{2}\right)=\sum_{k, m=1}^{\infty} H_{q, k, m}$ and which is of the

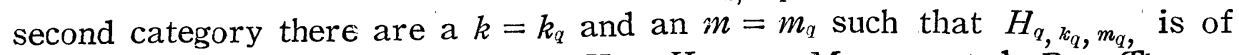
the second category. Denoting $H_{q}=H_{q}, k_{q}, m_{q}, \quad M_{q}=m_{7}$ and $P_{q}=T^{k_{q}}$ we see readily that (1.1) is proved. 
Now, for any fixed positive integer $m$ we have

(1.3) $\quad \underset{t}{\mathrm{E}}\left[t \in P_{q} ; \sup _{n}\left|S_{n}(x, t)\right| \geqq M_{q}\right] \supset \underset{t}{\mathrm{E}}\left[t \in P_{q} ; \max _{n<m}\left|S_{n}(x, t)\right| \geqq M_{q}\right]$.

If for a fixed $t$ the ineqnality

$$
\max _{n<m}\left|S_{n}(x, t)\right| \geqq M_{q}
$$

holds for every $x \in \mathrm{H}_{q}$, then (1.4) remains true for every $x \in H_{q}$ in virtue of the property $(a)$. Hence by (1.1) and (1.3) we have

$$
\text { meas } \underset{t}{\mathrm{E}}\left[t \in P_{q} ; \max _{n<m}\left|S_{n}(x, t)\right| \geqq M_{q}\right]<\frac{1}{q^{2}} \quad\left(x \in H_{q}\right) .
$$

Making $m \rightarrow \infty$ in (1.5) we deduce that

$$
\text { meas } \underset{t}{\mathrm{E}}\left[t \in P_{q} ; \sup _{n}\left|S_{n}(x, t)\right| \geqq M_{q}\right] \leqq \frac{1}{q^{2}} \quad\left(x \in H_{q}\right) .
$$

Since the set $\bar{H}_{q}$ is closed and of the second category, it contains a sphere $C_{q}$ of center $x_{v}=\left\{\gamma_{i}\right\}$ say. By the property of the distance in $E$, we may find a positive integer $j$ such that the coincidence of the first $j$ terms of $x_{0}$ and $x \in E$ implies $x \in C_{0}$. From the property $(b)$ of $S_{n}(x, t)$ by putting $\delta=1 / q^{2}$ we see that if $x^{\prime}$ and $x^{\prime \prime} \in E$ are $j$-equal, then there exist a set $J_{q} \subset I$ and a positive number $K_{q}>0$ such that

$$
\begin{aligned}
\mid S_{n}\left(x^{\prime} ; t\right)- & S_{n}\left(x^{\prime \prime}, t\right) \mid<K_{q} \\
\text { meas } J_{q}>\operatorname{meas} I-1 / q^{2} . & \left(n=1,2, \cdots ; t \in J_{q}\right),
\end{aligned}
$$

We shall prove next that

$$
\text { meas } \underset{t}{\mathrm{E}}\left[t \in P_{q} ; \sup _{w}\left|S_{n}(x, t)\right| \geqq K_{q}+M_{q}\right] \leqq \frac{2}{q^{2}} \quad(x \in E) .
$$

Let $t$ be contained in the set of the left-hand side of (1.9) for an $x_{1}=$ $\left\{\beta_{i}\right\} \in E$. Since $x_{0}$ and $x_{2}=\left\{\gamma_{1}, \cdots, \gamma_{j}, \beta_{j+1}, \cdots\right\}$ have the same first $j$ terms we have $x_{2} \in C_{q} \subset \bar{H}_{q}$. And if further $t \in P_{q} J_{q}$ we have by (1.7)

Hence we deduce that

$$
\begin{aligned}
\sup \left|S_{n}\left(x_{2}, t\right)\right| & \geqq \sup _{n}\left|S_{n}\left(x_{1}, t\right)\right|-\sup _{n}\left|S_{n}\left(x_{1}, t\right)-S_{n}\left(x_{2}, t\right)\right| \\
& \geqq\left(K_{q}+M_{q}\right)-K_{q}=M_{q} \quad(n=1,2, \cdots) .
\end{aligned}
$$

$$
\begin{aligned}
\underset{t}{\mathrm{E}}[t & \left.\in P_{q} ; \sup _{n}\left|S_{n}\left(x_{1}, t\right)\right| \geqq K_{q}+M_{I}\right] \\
& \subset \underset{t}{\mathrm{E}}\left[t \in P_{q} J_{q} ; \sup _{u}\left|S_{n}\left(x_{1}, t\right)\right| \geqq K_{q}+M_{\tau}\right]+\left(P_{q}-J_{q}\right) \\
& \subset \underset{t}{\mathrm{E}}\left[t \in P_{q} ; \sup _{n}\left|S_{n}\left(x_{2}, t\right)\right| \geqq M_{I}\right]+\left(P_{q}-J_{q}\right) .
\end{aligned}
$$

Each set of the last hand side being of measure $<1 / q^{2}$ by $(1.5)$ and $(1.8$ ), we get (1.9).

Now let us put

$$
A=\lim _{q} \sup P_{q}=\prod_{k=1}^{\infty} \sum_{q=k}^{\infty} P_{q} .
$$

To any $\eta>0$ there correspond two integers $k_{0}$ and $s_{0}$ such that

$$
\sum_{q=k_{0}}^{\infty} \frac{2}{q^{2}}<\frac{\eta}{2}, \quad \text { meas }\left(\sum_{q=k_{0}}^{\infty} P_{q}-\sum_{q=k_{0}}^{s_{0}} P_{q}\right)<\frac{\eta}{2} .
$$

By (1.9) there is a constant $L>0$ such that 


$$
\text { meas } \underset{t}{\mathrm{E}}\left[t \in P_{q} ; \sup _{n}\left|S_{n}(x, t)\right| \geqq L\right]<\frac{2}{q^{2}}
$$

$(x \in E)$, for $q=k_{0}, k_{0}+1, \cdots, s_{0}$. Finally from (1.11) and (1.12) we have for a fixed $x \in E$,

$$
\begin{aligned}
& \text { meas } \underset{t}{\mathrm{E}}\left[t \in A ; \sup _{n}\left|S_{n}(x, t)\right| \geqq L\right] \\
\leqq & \operatorname{meas} \underset{t}{\mathrm{E}}\left[t \in \sum_{q=k_{0}}^{\infty} P_{q} ; \sup _{n}\left|S_{n}(x, t)\right| \geqq L\right] \\
\leqq & \left.\operatorname{meas} \underset{t}{\mathrm{E}}\left[t \in \sum_{q=k_{0}}^{s_{0}} P_{q} ; \sup _{n} \mid S_{n} \cdot x, t\right) \mid \geqq L\right]+\operatorname{meas}\left(\sum_{q=k_{0}}^{\infty} P_{q}-\sum_{q=k_{0}}^{s_{0}} P_{q}\right) \\
\leqq & \sum_{q=k_{0}}^{s_{0}} \operatorname{meas} \underset{t}{\mathrm{E}}\left[t \in P_{q} ; \sup _{n}\left|S_{n}(x, t)\right| \geqq L\right]+\frac{\eta}{2} \\
\leqq & \sum_{q=k_{0}}^{s_{0}} \frac{2}{q^{2}}+\frac{\eta}{2}<\eta,
\end{aligned}
$$

which proves (i).

To prove (ii), suppose, if possible, that there exists a set $H \subset E$ of the second category such that $\left\{S_{n}(x, t)\right\}$ is bounded in measure on a set $Q(x) \subset I-A$ of positive measure. Then from (i) the sequence $\left\{S_{n}(x, t)\right\}$ is bounded in measure on $A+Q(x)$ tor all $x \in H$. Since meas $[A+Q(x)]$ $>$ meas $A \geqq \alpha_{0}$ we have

$$
H=\sum_{n=1}^{\infty} H_{n}, \quad H_{n}=\underset{x}{\mathrm{E}}\left[x \in H ; \text { meas }[A+Q(x)] \geqq \alpha_{0}+\frac{1}{n}\right] .
$$

And all the sets $H_{n}$ being of the first category by the definition of $\alpha_{0}$, these equalities contain a contradiction,

2. The $x$-set $E$ and $t$-set $I$ will be assumed the same as in the previous $\S$.

THEOREM 2. In the hypothesis of Theorem 1 let us replace the property (b) by the following

(c) if $x \in E$ and $x^{\prime} \in E$ are j-equal for some $j>0$, then for almost all $t \in I$ the sequences $\left\{S_{n}(x, t)\right\}$ and $\left\{S_{n}\left(x^{\prime}, t\right)\right\}$ have the same oscillation.

Then there exists a measurable set $B \subset I$ such that

(i) $\left\{S_{n}(x, t)\right\}$ is convergent almost everywhere on $B$ for all $x \in E$; and (ii) for all $x \in E$ except for $a$ set of the first category, $\left\{S_{n}(x, t)\right\}$ is divergent almost everywhere on $I-B$.

PROOF. For $x \in E$ let $\Gamma(x) \subset I$ denote the set of $t \in I$ for which $\left\{S_{n}(x, t)\right\}$ converges. Let $\delta_{0}$ be the least upper bound of $\delta>0$ such that the set $H(\delta)=\underset{x}{\mathrm{E}}[x \in E$; meas $\Gamma(x)>\delta]$ is of the second category. We may suppose that $\delta_{0}>0$.

For any positive integer $q$ and $k$, denoting by $H_{q, k}$ the set of all $x \in H\left(\delta_{0}-1 / q^{2}\right)$ for which meas $T_{k}>\delta_{0}-1 / q^{2}$ and meas $\left(T_{k}-T_{k} \Gamma(x)\right)<$ $1 / q^{2}$, we have $H\left(\delta_{0}-1 / q^{2}\right)=\sum_{k=1}^{\infty} H_{q}, T_{k}$. Then for some $k=k_{q}$ the set $H_{q, k_{q}}$ 
is of the second category. Put $H_{q}=H_{q}, k_{q}$ and $P_{q}=T_{k^{q}}$, we have then

$$
\text { meas }\left(P_{q}-P_{q} \Gamma(x)\right) \leqq 1 / q^{2}
$$

$\left(x \in H_{q}\right)$.

we shall prove that (2.1) remains true for any $x \in E$.

If $x \in H_{q}$ then for any $\eta>0$ there corresponds an integer $N=N(\eta, x)$ such that

$$
\text { meas } \underset{t}{\mathrm{E}}\left[t \in P_{q} ; \sup _{m, n>N}\left|S_{n}(x, t)-S_{m}(x, t)\right|>\eta\right]<\eta+\frac{1}{q^{2}} .
$$

In fact, the left-hand side of (2.2) is not greater than

$$
\text { meas } \underset{t}{\mathrm{E}}\left[t \in P_{q} \Gamma(x) ; \sup _{m, n>N}\left|S_{n}(x, t)-S_{m}(x, t)\right|>\eta\right]+\frac{1}{q^{2}}<\eta+\frac{1}{q^{2}},
$$

if $N$ is large enough.

Let $G_{q},{ }_{N}$ denote the set of all $x \in H_{q}$ for which (2.2) holds for a fixed $N$. Since $H_{1}=\sum_{N=1}^{\infty} G_{q, N}$ and which is of the second category, there is an $N_{q}=N_{q}(\boldsymbol{\eta})$ such that $G_{q}, N_{q}$ is of the second category. Denote $G_{q}=G_{q}, N_{q}$, We obtained :

(2.3) meas $\mathrm{E}_{t}\left[t \in P_{q} ; \sup _{m, n>N_{q}} S_{n}(x, t)-S_{m}(x, t) \mid>\eta\right]<\eta+\frac{1}{q^{2}} \quad\left(x \in G_{q}\right)$.

For $x_{0} \in G_{q}^{\prime}$ there exists an integer $M_{q}=M_{q}\left(x_{0}, \eta\right)$ such that

$$
\begin{aligned}
& \text { meas } \mathrm{E}_{t}\left[t \in P_{q} ; \sup _{m, n>N_{q}} S_{n}\left(x_{0}, t\right)-S_{m}\left(x_{0}, t\right) \mid>3 \eta\right] \\
&<\text { meas } \mathrm{E}_{t}\left[t \in P_{q} ; \sup _{m, n>N_{q}} S_{n}\left(x_{0}, t\right)-S_{m}\left(x_{1}, t\right) !>3 \eta\right]+\eta .
\end{aligned}
$$

By the property $(a)$ of $S ،(x, t)$, there exist an $x_{1} \in G_{l}$ and a set $J \subseteq I$ such that

$$
\sup _{M_{q}>n} \mid S_{n}\left(x_{0}, t\right)-S_{n}\left(x_{1}, t\right) i<\eta \quad(t \in J),
$$

On the other hand, if the inequality

$$
\sup _{x_{q}>n, n>N_{q}}\left|S_{n}\left(x_{i}, t\right)-S_{m}\left(x_{0}, t\right)\right|>3 \eta
$$

holds for all $t \in P_{q} J$, then by (2.5)

$$
\begin{aligned}
& \sup _{I_{q}>n, n>N_{q}}\left|S_{n}\left(x_{1}, t\right)-S_{m}\left(x_{1}, t\right)\right| \geqq \sup _{M_{q}>n, n>N_{p}}\left|S_{n}\left(x_{0}, t\right)-S_{n}\left(x_{0}, t\right)\right| \\
& \quad-\sup _{I_{q}>n>N_{q}}\left|S_{n}\left(x_{0}, t\right)-S_{n}\left(x_{1}, t\right)\right|-\sup _{M_{q}>n \rightarrow N_{q}}\left|S_{n}\left(x_{6}, t\right)-S_{m}\left(x_{1}, t\right)\right| \\
& \geqq 3 \eta-\eta-\eta=\eta .
\end{aligned}
$$

Next, we have

:2.8, $\quad$ meas $\mathrm{E}_{t}\left[t \in P_{q} ; \sup _{m, n>N_{q}}\left|S_{n}\left(x_{0}, t\right)-S_{m}\left(x_{0}, t\right)\right| \leqq 3 \eta\right]$

$$
\begin{aligned}
& \leqq \text { meas } \underset{t}{\mathrm{E}}\left[t \in P_{q} J ; \sup _{H_{q}>m, n>N_{g}}\left|S_{n}\left(x_{0}, t\right)-S_{m}\left(x_{1}, t\right)\right|>3 \eta\right]+2 \eta \\
& \text { (by (2.4), (2.6)) } \\
& \leqq \text { meas } \underset{t}{\mathrm{E}}\left[t \in P_{q} J ; \sup _{x_{u_{i}}>m, n>N_{q}}\left|S_{n}\left(x_{1}, t\right)-S_{m}\left(x_{1}, t\right)\right|>\eta\right]+2 \eta \\
& \leqq \text { meas } \mathrm{F}_{t}\left[t \in P_{q} ; \sup _{m, \iota>N_{q}}\left|S_{n}\left(x_{1}, t\right)-S_{m}\left(x_{1}, t\right)\right|>n\right\urcorner+2 n \text {. }
\end{aligned}
$$


From (2.3) and (2.8) we have

(2.9) meas $\underset{t}{\mathrm{E}}\left[t \in P_{q} ; \sup _{m, n>N q}\left|S_{n}\left(x_{0}, t\right)-S_{m}\left(x_{0}, t\right)\right|>3 \eta\right]<3 \eta+\frac{1}{q^{2}} \quad\left(x_{0} \in \bar{G}_{q}\right)$, where $N_{t}=N_{q}(\eta)$ and $G_{q}=G_{q}(\eta)$.

The set $G_{q}$ being closed and of the second category, it contains a sphere $C_{q}$; and then for any $x^{\prime} \in E$ there is an $x^{*} \in C_{q}$ which is $j$-equal to $x^{\prime}$ for some $j$. By the property $(c)$ of $S_{n}(x, t)$ and $(2.9)$ we deduce that

$$
\begin{aligned}
& \text { meas } \underset{t}{\mathrm{E}}\left[t \in P_{q} ; \limsup _{m, n \rightarrow \infty}\left|S_{n}\left(x^{\prime}, t\right)-S_{m}\left(x^{\prime}, t\right)\right|>3 \eta\right] \\
= & \text { meas } \underset{t}{\mathrm{E}}\left[\mathrm{t} \in P_{q} ; \limsup _{m, n \rightarrow \infty}\left|S_{n}\left(x^{*}, t\right)-S_{m}\left(x^{*}, t\right)\right|>3 \eta\right]<3 \eta+\frac{1}{q^{2}} .
\end{aligned}
$$

This relation means, $\eta$ being arbitrary, that for every $x \in E$ the sequence $\left\{S_{n}(x, t)\right\}$ converges for all $t \in P_{q}$ except for a set of measure $\leqq 1 / q^{2}$; that is, (2.1) holds for all $x \in E$.

$B=\lim \operatorname{suplet}_{q} P_{q}$, then as we see immediately, the sequence $\left\{\boldsymbol{S}_{n}(\boldsymbol{x}, t)\right\}$ converges almost everywhere on $B$ for all $x \in E$, which proves (i).

As in the proof of (ii) of Theorem 1, we may prove (ii) of Theorem 2 by the inequalities meas $B \geqq \lim _{q \rightarrow \infty}$ sup meas $P_{q} \geqq \delta_{0}$,

q. e. d.

3. For the application of our theorems to the theory of series, we shall define some metric spaces whose elements are sequences of real numbers. They are:

$\Omega_{1}$ : space of all the sequences of positive numbers which tend to zero and are not increasing from some range on. The distance of the elements $x=\left\{\beta_{i}\right\}$ and $x^{\prime}=\left\{\gamma_{i}\right\}$ is $\rho\left(x, x^{\prime}\right)=\sup _{i}\left|\beta_{i}-\gamma_{i}\right|$.

$\Omega_{2}$ : space of all the sequences of real numbers which tend to zero. The distance of $x=\left\{\beta_{i}\right\}$ and $x^{\prime}=\left\{\gamma_{i}\right\}$ is $\rho\left(x, x^{\prime}\right)=\sup _{i}\left|\beta_{i}-\gamma_{i}\right|$.

$\Omega_{3}$ : space of all the sequences of positive integers $\left\{n_{i}\right\}$ such that $n_{1}=1$ and $\lim _{i \rightarrow \infty}\left(n_{i}-n_{i-1}\right)=+\infty$. The distance of $x=\left\{n_{i}\right\}$ and $x^{\prime}=\left\{m^{\prime}\right\}$ is

$$
\rho\left(x, x^{\prime}\right)=\sum_{i=1}^{\infty} \frac{1}{2^{i}} \frac{|| n_{i+1}-n_{i}|-| m_{i+1}-m_{i}||}{|| n_{i+1}-n_{i} \mid} .
$$

$\Omega_{4}$ : space of all the permutations of all the positive integers and a finite number of arbitrary positive integers. The distance of $x=\left\{\boldsymbol{n}_{i}\right\}$ and $x^{\prime}=\left\{m_{i}\right\}$ is

$$
\rho\left(x, x^{\prime}\right)=\sum_{i=1}^{\infty} \frac{1}{2^{i}} \frac{\left|n_{i}-m_{i}\right|}{1+\left|n_{i}-m_{i}\right|} .
$$

$\Omega_{5}$ : space of all the sequences which consist cf infinite number of 0 's and infinite number of 1 's. For any $x \in \Omega_{5}$, there corresponds a dyadic irrational in $(0,1)$ whose coefficients of dyadic expansion is $x$. The distance is defined as a usual distance for the corresponding dyadic irrationals.

$\Omega_{6}$ : space of all the sequences which consist of infinite number of +1 's and that of -1 's. Let $\left\{\boldsymbol{r}_{n}(u)\right\}$ be the Rademacher system $(0<u<1)$. then, to any $x \in \Omega_{6}$ there corresponds a $u, 0<u<1$ such that $x=\left\{\boldsymbol{r}_{i}(\boldsymbol{u})\right\}$. 
The distance is defined as an ordinary distance in $(0,1)$ for the corresponding $u$ 's.

The spaces thus defined are all of the second category, in fact $\Omega_{1}, \Omega_{2}$ and $\Omega_{3}$ are complete metric space; for $\Omega_{4}$ this is proved by R. P. Agnew [\%.] and for $\Omega_{5}$ and $\Omega_{j}$ this fact is obvious. If $x=\left\{\gamma_{i}\right\} \in \Omega_{\lambda}$ and $\left\{\boldsymbol{\beta}_{i}\right\} \in \Omega_{\lambda}$ then for every $j, x_{j}=\left\{\gamma_{1}, \cdots, \gamma_{i}, \beta_{j+1}, \cdots\right\} \in \Omega_{\lambda}$ and $x_{j} \rightarrow x$ as $j \rightarrow \infty$ $(\lambda=1,2, \cdots, 6)$.

Let $\sum_{i=1}^{\infty} a_{i}(t)$ be a series whose tems are finite measurable functions defined in a measurable set $I$ of measure $>0$ and $<\infty$ we shall consider the following two cases:

( I ) $\quad \limsup _{n \rightarrow \infty}\left|\sum_{i=1}^{\infty} a_{i}(t)\right|=\infty \quad$ for almost all $t \in \mathrm{I}$,

(II) the series $\sum_{i=1}^{\infty} a_{i}(t)$ diverges almost everywhere in $I$.

Evidently the case (I) is a special case of (II).

In the case (I) we have the following results.

LEMMA 1. (See $[\tilde{y}]$ ). There exists an $x=\left\{\lambda_{i}\right\} \in \Omega_{1}$ such that $\limsup _{n \rightarrow \infty}\left|\sum_{i=1}^{n} \lambda_{i} a_{i}(t)\right|=\infty \quad$ for almost all $t \in I$.

Lemma 2. (See [5]). There exists an $x=\left\{n_{i}\right\} \in \Omega_{3}$ such that $\limsup _{m \rightarrow \infty}\left|\sum_{i=1}^{m} a_{i i_{i}}(t)\right|=\infty \quad$ for almost all $t \in I$.

LEMMA 3. There exists a dyadic irrationa! $u=0, \beta_{1} \beta_{2} \cdots$ (dyadic expansion) such that

$$
\limsup _{n \rightarrow \infty}\left|\sum_{i=1}^{n} \beta_{i} a_{i}(t)\right|=\infty \quad \text { for almost all } t \in I .
$$

LEMMA 4. There exists a dyadic irrational $u$ such that

$$
\limsup _{n \rightarrow \infty}\left|\sum_{i=1}^{n} r_{i}(u) a_{i}(t)\right|=\infty \quad \text { for almost all } t \in I .
$$

The proof of Lemmas 3 and 4 may be done as in the paper [5].

In the case (II) we have the following two lemmas.

LEMMA 5. There exists a dyadic irrational $u=0, \beta_{1} \beta_{2} \cdots$ (dyadic expansion) such that $\Sigma \beta_{i} a_{i}(t)$ diverges a!most everywhere in $I$.

LEMMA 6. There exists a dyadic irrational $u$ such that $\Sigma r_{i}(u) a_{i}(t)$ diverges almost everywhere in $I$.

PROGF OF LEMMA 5. for a positive integer $p$, let $I_{p}$ denote the set of all $t \in I$ for which 
We have evidently

$$
\limsup _{m, n \rightarrow \infty}\left|\sum_{i=n}^{m} a_{i}(t)\right| \geqq \frac{1}{p} .
$$

$$
I=\sum_{p=1}^{\infty} I_{p}
$$

We can find a positive integer $n_{1}$ and a set $I_{1}^{(1)}$ such that

$$
\begin{gathered}
I_{1}^{(1)}=\mathrm{E}_{t}^{\mathrm{E}}\left[t \in I_{1} ; \sup _{n_{1} \geqq i \geqq j \geqq 1}\left|a_{j}(t)+\cdots+a_{i}(t)\right| \geqq 1_{-}^{-},\right. \\
\text {meas } I_{1}^{(1)}>\text { meas } I_{-}-1 / 2 ;
\end{gathered}
$$

and then we can find an $n_{: 2}>n_{1}+1$ and an $I_{2}^{(1)}$ such that

$$
\begin{gathered}
I_{2}^{(1)}=\underset{t}{\mathrm{E}}\left[t \in I_{2} ; \sup _{n \cong i \geqq j>n_{1}+1}\left|a_{j}(t)+\cdots+a_{i}(t)\right| \geqq-\frac{1}{2}\right], \\
\text { meas } I_{2}^{(1)}>\text { meas } I_{2}-1 / 2 ;
\end{gathered}
$$

next we can find $n_{3}>n_{2}+1$ and $I_{1}^{(2)}$ such that

$$
\begin{gathered}
I_{1}^{(2)}=\mathrm{E}\left[t \in I_{1} ; \sup _{n_{3} \geqq i \geqq j>n_{2}+1}\left|a_{j}(t)+\cdots+a_{i}(t)\right| \geqq 1\right], \\
\text { meas } I_{1}^{(2)}>\text { meas } I_{1}-1 / 2^{2} .
\end{gathered}
$$

Similarly we shall define $I_{\nu}^{(\nu)}(p, \nu=1,2, \cdots)$ and $n_{s}(s=1,2, \cdots)$ considering $I_{1} ; I_{2}, I_{1} ; I_{3}, I_{2}, \cdots$ successively. That is, in the $s \equiv \frac{1}{2}\left[\nu^{2}+(2 p-1) \nu\right.$ $+(p-1)(p-2)]$-th step we treat the set $I_{p}$ for every $\nu$; if $n_{s-1}$ was defined we may find a positive integer $n_{s}$ and a set $I_{p}^{(\nu)}$ such that

$$
\begin{aligned}
I_{p}^{(v)}= & \underset{t}{\mathrm{E}}\left[t \in I_{p} ; \sup _{n_{s} \geqq \geqq j>n_{s-1}+1}\left|a_{j}(t)+\cdots+a_{i}(t)\right| \geqq \frac{1}{p}\right], \\
& \text { meas } I_{p}^{(\nu)}>\text { meas } I_{p}-1 / 2^{\nu} .
\end{aligned}
$$

The double sequence $\left\{I_{p}^{(\nu)}\right\}$ and the sequence $\left\{n_{s}\right\}$ were thus defined by induction.

Let now $\beta_{i}=0$ if $i$ is of the form $n_{s}+1$ and let $\beta_{i}=1$ otherwise. Then the number $u=0, \beta_{1} \beta_{2} \cdots$ is evidently dyadically irrational.

We shall show that the series

$$
\sum_{i=1}^{\infty} \beta_{i} a_{i}(t)
$$

diverges almost everywhere in $I$. Let $I_{p}^{*}=\lim \inf _{\nu} I_{p}^{(v)}$ and $I^{*}=\sum_{p=1}^{\infty} I_{p}^{*}$, then by (3.6) and (3.1) meas $I_{i}^{*}=$ meas $I_{i n}$ and meas $I=$ meas $I^{*}$.

If $t \in I^{*}$ we have $x \in I_{j}^{*}$ for a cartain $p$, and $x \in I_{p}^{(v)}$ for all sufficiently large $\nu$; hence by the definition of $I_{p}^{(\nu)}$ the series (3.7) has the oscillation $\geqq 1 / p$, that is (3.7) diverges in $I^{*}$,

The proof of Lemma 6 may be done similarly: suppose that $\left\{n_{s}\right\}$ is defined as above. let $\varepsilon_{i}=-1$ if $i$ is of the form $n_{s}+1$ and $\varepsilon_{i}=+1$ otherwise. Let $u$ be such that $\left\{\boldsymbol{r}_{i}(u)\right\}=\left\{\varepsilon_{i}\right\}$, then $u$ is dyadically irrational. The series $\sum \varepsilon_{i} a_{i}(t)$ diverges almost everywhere in $I$ 
Let us define $\left\{S_{n}(x, t)\right\}$.

For $x=\left\{\lambda_{i}\right\} \in \Omega_{1}$ or $\Omega_{2}$, put

$$
S_{n}(x, t)=\sum_{i=1}^{n} \lambda_{i} a_{i}(t)
$$

For $x=\left\{n_{i}\right\} \in \Omega_{3}$, put

$$
S_{n}(x, t)=\sum_{n_{i}<n} a_{n_{i}}(t) .
$$

For $x=\left\{n_{i}\right\} \in \Omega_{4}$, put

$$
S_{n}(x, t)=\sum_{i=1}^{n} a_{n_{i}}(t) .
$$

For $x=\left\{\beta_{i}\right\} \in \Omega_{5}$ or $\Omega_{6}$, put

$$
S_{n}(x, t)=\sum_{i=1}^{n} \beta_{i} a_{i}(t)
$$

The sequence $\left\{S_{n}(x, t)\right\}$ thus defined satisfies the condition (a) and (c) of Theorems 1 and 2 taking $E=\Omega_{\mu}(\mu=1,2, \cdots, 6)$. If $x$ and $x^{\prime} \in \Omega_{\mu}$ $(\mu \neq 4)$ are $j$-equal we see

$$
\left.\left|S_{n}(x, t)-S_{n}\left(x^{\prime} ; t\right)\right| \leqq K_{1} \sum_{i=1}^{j} \mid a_{i}(t)\right\} \quad\left(K_{1}: \text { constant }\right),
$$

and the condition $(b)$ is easily verified. If $\left|a_{i}(t)\right|<K_{2}$ for all $i=1,2, \cdots$ and $t \in I$, then $(b)$ is also satisfied for $\Omega_{4}$.

Now applying Theorem 1 we obtain the set $A \subset I$, and which cannot be of measure positive in the case (I). In fact, if meas $A \geqq \alpha_{0}>0$, then by (i) of Theorem 1 there is an $L>0$ such that

(3.13) meas $\underset{t}{\mathrm{E}}\left[t \in A ; \sup _{n}\left|S_{13}(x, t)\right| \geqq I\right]<\frac{\alpha_{0}}{2}$ for all $x \in \Omega_{\mu}$.

On the other hand by Lemmas $1,2,3$, or 4 there is an $x_{0} \in \Omega_{\mu}$ such that (3.14) $\limsup _{n \rightarrow \infty}\left|S_{n}\left(x_{0}, t\right)\right|=\infty$ for almost all $t \in I$.

Evidently (3.13) and (3.14) make a contradiction, hence we have meas $A=0$.

Similarly applying Theorem 2 for $E=\Omega_{4}, \Omega_{5}$ and $\Omega_{6}$, we obtain $B \subset I$, and which is of measure zero in the case (II).

When meas $I=\infty$, let $I=\sum_{j=1}^{\infty} I_{j}$, meas $I_{j}<\infty$ and discussing as above on each $I_{j}$ we obtain the following theorem.

THEOREM 3. Let $\sum_{i=1}^{\infty} a_{i}(t)$ be a series whose terms are finite measurable functions defined in a measurable set $I$ of measure $>0$.

Then in the case (I), for all $x \in \Omega_{\mu}\left(\mu=1,2, \cdots, 6\right.$; for $\Omega_{4}$ suppose that $\left|a_{i}(t)\right|<K_{2}$ for all $i=1,2, \cdots$, , and $t \in I$ ) except for a set of the first category, the modified series with partial sum $S_{n}(x, t)$ is divergent to $\pm \infty$ or oscillates infinitely for almost all $t \in I$.

And in the case (II), for all $x \in \Omega_{\mu}(\mu=4,5,6)$ except for a set of the 
first category, the modified series with partial sum $S_{\mu}(x, t)$ is divergent for almost all $t \in I$.

REMARK. Let $T$ be a Toeplitz summation method. Then the analogue of Theorem 3 holds concerning the $T$-mean $\sigma_{n}(x, t)$ of the series instead of $S_{n}(x, t)$.

In the second part of Theorem 3 we cannot replace the condition (II) by the condition that the series is not absolutely convergent almost everywhere. Indeed, the series

$$
\sum_{n=1}^{\infty} \frac{\cos n t}{n}
$$

is not absolutely convergent for all $t \in(0,2 \pi)$, but the series

$$
\begin{array}{lr}
\sum_{k} \frac{\cos n_{k} t}{n_{k}} & \left(x=\left\{n_{k}\right\}\right), \\
\sum_{n} \beta_{n} \cos n t & \left(x=\left\{\beta_{n}\right\}\right), \\
\sum_{n} r_{n}(u) \frac{\cos n t}{n} & \left(x=\left\{r_{n}(u)\right\}\right)
\end{array}
$$

are all convergent almost everywhere in $(0,2 \pi)$ for every $x \in \Omega_{\mu}(\mu=4$, 5,6 respectively).

\section{REFERENCES}

1. Acinew, R. P., Subseries of series which are not absulitely convergent, Bull. Amer. Math. Sic., 53(1947) 118-120.

2. Linew, R. P., On rearrangement of series, Bull. Amer. Math. Suc., 46(1940)797-799.

3. Hit., I. D., S'me theorems on subseries, Ball. Imer. Math. Soc,; 48(1942)103-108.

4. SAks, S., On some functionals, II., Trans. Imer. Math. Soc., 41(1937) 160-170.

5. Tsuchikuna, T., On some divergence problems, Tôhoku Math. Journ., 2(1950)30-39.

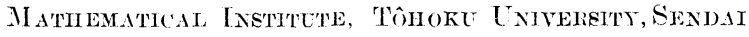

